\title{
ON-ROAD DRIVING ASSESSMENT ERRORS ASSOCIATED WITH PASS AND FAIL OUTCOMES FOR OLDER DRIVERS WITH COGNITIVE IMPAIRMENT
}

\author{
Petra Hoggarth ${ }^{1,2}$, Carrie Innes ${ }^{1,3}$, John Dalrymple-Alford ${ }^{1,2,4}$, \& Richard Jones ${ }^{1,2,3,4,5}$ \\ ${ }^{1}$ Van der Veer Institute for Parkinson's \& Brain Research \\ ${ }^{2}$ Department of Psychology, University of Canterbury \\ ${ }^{3}$ Department of Medical Physics \& Bioengineering, Christchurch Hospital \\ ${ }^{4}$ Department of Medicine, University of Otago \\ ${ }^{5}$ Department of Electrical \& Computer Engineering, University of Canterbury \\ Christchurch, New Zealand \\ E-mail: petra.hoggarth@vanderveer.org.nz
}

\begin{abstract}
Summary: Most on-road assessments do not make use of standardized scoring to determine driver safety. The current study sought to find a subset of driving errors that were related to on-road Pass and Fail outcomes in a group of 60 older drivers with cognitive impairment, and that were also considered important contributors to a Fail outcome by the driving specialist occupational therapist who administered the assessment. A number of useful errors were found that suggest that even a non-standardized driving assessment could incorporate a short list of driving errors that may assist in the determination of driving ability.
\end{abstract}

\section{INTRODUCTION}

On-road driving assessment is a commonly used tool to determine whether a driver with cognitive impairment or dementia is safe to continue driving. Some standardized assessments have been tested for inter-rater and test-retest reliability, with the former usually found to be moderate to high, and the latter in the moderate range (Hagge, 1994; Fitten et al., 1995; Romanowicz \& Hagge, 1995; Hunt et al., 1997; Janke \& Eberhard, 1998). Unfortunately, standardized on-road driving assessments are often not used as part of routine driving assessment. Korner-Bitensky et al. (2006) surveyed the driving assessment methods of 144 American and Canadian driving assessors. Ninety-four percent of respondents reported routinely using on-road assessments as part of their evaluation, yet only $24 \%$ used a standardized scoring system, and only $10 \%$ used a pre-defined cutoff score to define driving competency. Only two respondents reported using a standardized road test. The use of un-standardized on-road assessments in naturalistic research samples can make results difficult to generalize. Likewise, applying the results of studies that use standardized on-road assessments and scoring to realworld assessment practices can be equally as difficult.

The appeal of a non-standardized format is in the maximum flexibility it allows an assessor to draw on their often considerable clinical experience in deciding whether a person is a safe driver. For example, a standardized route cannot be used when an assessment is performed from a person's home and in their local area. A standardized on-road scoring system does not record all variables that may figure into an assessor's decision-making process, such as missed appointments that may indicate a memory problem. This flexibility in assessment may be particularly important when assessing people with cognitive impairment or dementia due to the idiosyncratic way impairments affect the abilities of individuals. We believe, however, that it is 
important that at least some standardization of on-road scoring is present in order that judgments made between drivers and between assessors are as equitable as possible.

For the current study, 60 drivers with suspected or diagnosed cognitive impairment or dementia were recruited. All completed an on-road driving assessment as used in New Zealand for assessing the impact of medical conditions on driving safety. This assessment did not use a standardized route or a predetermined rating of errors to determine a Pass or Fail outcome. Rather, the occupational therapist administering the assessment used a global decision-making process encompassing performance on the on-road assessment. The aim of the study was to find errors that occurred during the assessment that discriminated between those rated as on-road Pass and Fail. A detailed error list was constructed based on the results of a previous driving study. This was completed for each participant by both the driving instructor and occupational therapist who administered the assessment. Ideally, errors with a high degree of face validity to a driving assessor would be found that would be useful in discriminating between safe and unsafe drivers and could be used as part of the standard medical driving assessment. This would allow some form of objective scoring to be used in determining a Pass of Fail outcome for an assessment that currently does not have standardized scoring.

The study had three aims:

1. Determine the inter-rater agreement of commission of errors between the occupational therapist and driving instructor who administered the assessment.

2. Determine the errors most frequently cited by the occupational therapist as contributing to a Fail score.

3. Determine which errors discriminated between those who passed and failed the assessment and whether these errors were the same as those rated as most often contributing to a Fail score by the occupational therapist.

\section{METHODS}

\section{Participants}

Participants with a current full driver's licence were recruited from consecutive referrals to the Driving and Vehicle Assessment Service (DAVAS) at Burwood Hospital, Christchurch, New Zealand. Eligible referrals had suspected or diagnosed Alzheimer's dementia, memory or cognitive impairment listed as the reason for referral. These criteria were designed to include those participants who were likely to have Alzheimer's dementia or amnestic mild cognitive impairment.

Sixty participants were recruited: 36 males and 24 females with a mean age of 77.9 years $(\mathrm{SD}=$ 7.8, range 58-92). Participants gave informed consent. The study was approved by the Upper South A Regional Ethics Committee, Canterbury, New Zealand.

\section{Driving Error List}

A previous study investigated the on-road driving ability of 60 cognitively healthy older drivers (Hoggarth et al., 2010). An informal report including observed driving errors was compiled for 
each participant by the assessing occupational therapist. An error list with a standardized recording procedure was constructed based on the errors performed in this prior study. Noted errors were tallied and ranked, with the most common on-road errors forming the Driving Error List used for the current study. This list was given to the driving instructor of the prior study and two occupational therapists in order to add other errors that they commonly observed in people with dementia. Items in the final Driving Error List are presented in Table 1.

\section{Testing Procedure}

Participants completed an on-road driving assessment of around 45 minutes administered by an experienced driving occupational therapist and a driving instructor from the Driving and Vehicle Assessment Service at Burwood Hospital, Christchurch. Fifty-six drivers completed the on-road assessment in their own car, with the remaining four using an assessment centre car. The driving instructor sat in the passenger seat and provided directions for the driving route and maintained safety of the vehicle if required. The occupational therapist sat in the rear to assess driving behaviour. Twelve $(20 \%)$ participants started the drive from their own homes, with the remainder $80 \%$ starting from the Hospital. Assessment from home was made for a variety of different reasons such as availability of a family member in order to drive the participant home if they received a Fail outcome.

The driving instructor and the occupational therapist completed the Driving Error List independently at the end of each assessment. Assessors ticked one box to indicate which errors were performed during the assessment and another box if the error was cited as a reason for a Fail rating for the on-road driving performance. The assessors could also record additional errors as they arose.As was normal procedure, a global decision of Pass or Fail was made by the occupational therapist based on the entirety of the participant's performance during the assessment.

\section{Data Analysis}

Intra-class correlations were computed using Cronbach's Alpha to assess reliability between the two raters. Errors identified by the occupational therapist as contributing to on-road Fail were summed and ranked by frequency. The relationship between each error and Pass and Fail groups was investigated using Fisher's Exact Test (for expected cell frequencies $<5$ ). The Phi Coefficient was computed as a measure of association of each error to the Pass and Fail rating. The Phi Coefficient is equivalent to a Pearson's $r$ for a bivariate correlation.

\section{RESULTS}

Twenty-one of the 60 participants (35\%) received a Fail score on the on-road assessment. There was a trend for the Fail group to have a higher mean age than the Pass group (mean pass $=76.4$ years, mean fail $=80.8$ years, $Z=-1.88, p=.06$ ). There was no difference in sex between groups $($ male $=1$, female $=2$ : mean pass $=1.41$, mean fail $=1.38, Z=-.22, p=.83$ ), and no difference in Pass and Fail outcomes in those who started the on-road assessment from home versus the hospital $(Z=-.537, p=.59)$. Those who failed the on-road performed more on-road errors than those who passed, $($ Fail mean $=11.1$, Pass mean $=2.3, Z=-6.40, p<.001)$. 
Eight additional unique errors not found on the error list were recorded: 'Approached intersections at excessive speed' (7 participants), 'Incorrect use of turning bays' (3 participants), 'Failure to stop at stop sign' (3 participants), 'Braking and accelerating at the same time' (3 participants), 'Excessive acceleration' (1 participant), 'Went to wrong side of the road when approaching intersections' (1 participant), 'Late moving into turning lane' (1 participant), and 'Went through red light' (1 participant). Since these errors were not present on the error list for systematic rating they were not included in the following calculations, but may be useful to be added to a revised error list.

Table 1 displays the frequency that each error was rated by the two raters and Cronbach's alpha.

Table 1. Cronbach's alpha and $95 \%$ confidence interval for agreement on commission of errors between raters

\begin{tabular}{|c|c|c|c|c|}
\hline Type of Error & $\begin{array}{l}\text { Frequency } \\
\text { rated by } \\
\text { occupational } \\
\text { therapist }\end{array}$ & $\begin{array}{l}\text { Frequency } \\
\text { rated by } \\
\text { driving } \\
\text { instructor }\end{array}$ & $\begin{array}{l}\text { Cronbach's } \\
\text { alpha }\end{array}$ & $\begin{array}{c}95 \% \\
\text { confidence } \\
\text { interval }\end{array}$ \\
\hline Fails to follow pedestrian crossing rules & 3 & 3 & 1.00 & $1.00-1.00$ \\
\hline Decreased awareness of environment & 21 & 20 & 0.98 & $.97-.99$ \\
\hline Decreased awareness of other road users & 21 & 19 & 0.96 & $.94-.98$ \\
\hline Driving/starting in wrong gear & 7 & 6 & 0.96 & $.93-.97$ \\
\hline Inappropriate gap selection & 18 & 15 & 0.94 & $.98-.96$ \\
\hline Incorrect use of give way rules at intersections & 14 & 13 & 0.92 & $.87-.95$ \\
\hline Driving below the speed limit & 16 & 16 & 0.91 & $.84-.94$ \\
\hline Driving above the speed limit & 29 & 27 & 0.89 & $.82-.93$ \\
\hline Incorrect indication at a roundabout & 42 & 44 & 0.86 & $.77-.92$ \\
\hline Turned into incorrect lane on multi-lane road & 4 & 7 & 0.83 & $.72-.90$ \\
\hline Inappropriate use of arrow traffic lights & 2 & 1 & 0.80 & $.66-.88$ \\
\hline Driving too close to (or over) centre line & 9 & 11 & 0.78 & $.64-.87$ \\
\hline Lack of scanning techniques & 19 & 21 & 0.77 & $.62-.86$ \\
\hline Driving too close to (or over) left line & 15 & 11 & 0.76 & $.60-.86$ \\
\hline Didn't react in time to situation/Incorrect action taken & 13 & 8 & 0.76 & $.60-.86$ \\
\hline Lack of mirror use & 21 & 24 & 0.76 & $.60-.86$ \\
\hline Problem cornering: speed or position & 8 & 8 & 0.72 & $.54-.84$ \\
\hline Incorrect use of lanes in roundabout & 3 & 4 & 0.71 & $.51-.83$ \\
\hline Fails to observe signs & 8 & 9 & 0.69 & $.47-.81$ \\
\hline Immediate fail error (e.g. crash) & 2 & 7 & 0.61 & $.34-.77$ \\
\hline Lack of blind spot check & 17 & 23 & 0.59 & $.31-.75$ \\
\hline Following other cars too closely & 12 & 3 & 0.56 & $.26-.74$ \\
\hline Didn't apply 12 second search & 6 & 17 & 0.55 & $.24-.73$ \\
\hline Gear grinding/over-revving & 5 & 1 & 0.50 & $.16-.70$ \\
\hline Stopping too closely behind cars & 5 & 2 & 0.41 & $.01-.65$ \\
\hline Approaching intersections at excessive speed & 2 & 6 & 0.36 & $-.07-.62$ \\
\hline Incorrect indication at an intersection & 2 & 5 & -0.11 & $-.86-.34$ \\
\hline Incorrect indication for lane changes & 1 & 0 & N/A & $\mathrm{N} / \mathrm{A}$ \\
\hline Fails to give way to pedestrians at intersection & 1 & 0 & $\mathrm{~N} / \mathrm{A}$ & $\mathrm{N} / \mathrm{A}$ \\
\hline
\end{tabular}


There was a high degree of agreement between the raters with 11 error ratings with alphas of 0.80 or above. The error 'Incorrect indication at an intersection' had a negative alpha of -0.11 due to no agreement between the two ratings for any of the 7 participants who made this error.

The number of times an error was rated by the occupational therapist as contributing to a Fail score were ranked (Table 2). The two most frequently rated reasons for a Fail score were 'Decreased awareness of other road users' and 'Decreased awareness of environment', with this second reason being rated as a reason for all 21 participants who failed the assessment. The error 'Incorrect indication at a roundabout' had the highest occurrence in the sample as a whole (42 participants according to the occupational therapist's rating) but was only considered by the occupational therapist to have directly contributed to one person's Fail score.

Table 2. Frequency that errors were rated as contributing to a Fail outcome by the occupational therapist, and the statistical relationship of errors to Pass and Fail outcomes

\begin{tabular}{|c|c|c|c|}
\hline Error & $\begin{array}{c}\text { Frequency rated } \\
\text { as contributing } \\
\text { to the Fail score }\end{array}$ & $\begin{array}{c}\text { Fisher's Exact } \\
\text { Test } \\
p \text { value } \\
\end{array}$ & Phi coefficient \\
\hline Decreased awareness of other road users & 21 & $<.01$ & 1.00 \\
\hline Decreased awareness of environment & 20 & $<.01$ & 0.93 \\
\hline Lack of scanning techniques & 15 & $<.01$ & 0.78 \\
\hline Inappropriate gap selection & 13 & $<.01$ & 0.74 \\
\hline Driving above the speed limit & 13 & $<.01$ & 0.48 \\
\hline Incorrect use of give way rules at intersections & 12 & $<.01$ & 0.67 \\
\hline Didn't react in time to situation / incorrect action taken & 10 & $<.01$ & 0.55 \\
\hline Driving below the speed limit & 8 & 0.01 & 0.35 \\
\hline Driving too close to (or over) centre line & 7 & $<.01$ & 0.47 \\
\hline Driving too close to (or over) left line & 6 & $<.01$ & 0.54 \\
\hline Problem cornering: speed or position & 6 & $<.01$ & 0.53 \\
\hline Lack of mirror use & 6 & $<.01$ & 0.49 \\
\hline Lack of blind spot check & 5 & $<.01$ & 0.39 \\
\hline Following other cars too closely & 5 & 0.09 & 0.24 \\
\hline Gear grinding/over-revving & 3 & $<.01$ & 0.41 \\
\hline Didn't apply 12 second search & 3 & 0.02 & 0.34 \\
\hline Fails to observe signs & 3 & 0.02 & 0.33 \\
\hline Incorrect use of lanes in roundabout & 3 & 0.04 & 0.31 \\
\hline Stopping too closely behind cars & 3 & 0.05 & 0.28 \\
\hline Driving/starting in wrong gear & 2 & 0.23 & 0.17 \\
\hline Incorrect indication at an intersection & 1 & 0.12 & 0.25 \\
\hline Incorrect indication at a roundabout & 1 & 0.56 & 0.10 \\
\hline Fails to follow pedestrian crossing rules & 1 & 1.00 & -0.01 \\
\hline Immediate fail error (e.g. crash) & 0 & 0.10 & 0.25 \\
\hline Incorrect indication for lane changes & 0 & 0.35 & 0.18 \\
\hline Fails to give way to pedestrians at intersection & 0 & 1.00 & -0.10 \\
\hline Inappropriate use of arrow traffic lights & 0 & 0.54 & -0.14 \\
\hline Turned into incorrect lane on multi-lane road & 0 & 0.29 & -0.20 \\
\hline
\end{tabular}

Eighteen errors were related to Pass and Fail outcomes (Table 2). All but four variables were in the expected direction of occurring more frequently in the Fail group. Three variables did not occur in the Fail group at all: 'Turned into incorrect lane on multi-lane road', 'Fails to give way 
to pedestrians at intersection', and 'Inappropriate use of arrow traffic lights'. The error 'Fails to follow pedestrian crossing rules' occurred once in the Fail group and twice in the Pass group.

The errors most related to a Fail outcome are shown by the largest phi coefficients and are similar to the occupational therapist's self-rated contribution of error to Fail scores (Table 2). Of the top 10 errors rated as contributing to Fail scores by the occupational therapist, 8 were ranked in the top ten by phi coefficient for errors associated with Fail scores: 'Decreased awareness of other road users', 'Decreased awareness of environment', 'Lack of scanning techniques', 'Inappropriate gap selection', 'Incorrect use of give way rules at intersections', 'Didn't react in time to situation / incorrect action taken', 'Driving too close to (or over) left line', and 'Driving above the speed limit'.

\section{DISCUSSION}

Inter-rater agreement on the commission of driving errors was high for many items. This indicates that many of the listed errors were identified independently by each rater.

Discrepancies in some ratings could be due to the threshold of each rater in defining when an error occurred, and also due to the driving instructor's dual task of guiding the drive as well as maintaining safety of the vehicle, with the occupational therapist able to concentrate solely on driving behaviour.

The two measures most commonly cited as contributing to a Fail outcome were not errors per se, but rather a judgment made by the occupational therapist that a driver exhibited decreased awareness of the environment and/or other road users. The fact that these two awareness measures were rated for all but one Fail outcome suggests that a single measure of decreased awareness could be used instead of two separate ones. This result shows the importance of maintaining items which are not simply based on the performance of an error but rather on an interpretation based on a pattern of errors that indicate decreased awareness.

There was a high level of agreement between the subjective ratings of the occupational therapist as to which errors were especially important to a Fail outcome, and the degree to which the frequency of these errors (whether or not they were rated as important) were associated with onroad Fail scores. This suggests that a select choice of errors may be acceptable to assessors who prefer a flexible assessment procedure.

There are limitations to this study. Firstly, the occupational therapist may have been biased to recall more driving errors for drivers whom she had decided would receive Fail outcomes, thus the number of errors recalled may be inflated in the Fail group. The number of errors selected for the error list was limited and additional errors would likely have been useful, particularly errors more related to the types of difficulties that might be expected to be more common in drivers with cognitive impairment - confusion for example.

Finding ways to bridge the gap between researchers who ideally prefer a standardized on-road assessment and on-road assessors who frequently prefer a more flexible and less standardized approach could be achieved, in part, by including a checklist of errors that have been found to relate to on-road driving outcome in older adults with cognitive impairment. This study found a 
number of potentially useful error measures that were related to on-road Pass and Fail outcomes and were also rated as useful contributors to identifying people with on-road fail outcomes by an occupational therapist. Including an evidence-based error list as part of a non-standardized driving assessment would allow for systematic collection of error information that may contribute to more reliable Pass and Fail outcomes.

\section{ACKNOWLEDGEMENTS}

The authors would like to acknowledge funding for this project from the Canterbury Medical Research Foundation, Accident Compensation Corporation, Christchurch Neurotechnology Research Programme, the University of Canterbury, the Maurice and Phyllis Paykel Trust, and Universities New Zealand - Te Pōkai Tara.

\section{REFERENCES}

Fitten, L. J., Perryman, K. M., Wilkinson, C. J., Little, R. J., Burns, M. M., Pachana, N., Mervis, R., Malmgren, R., Siembieda, D. W., \& Ganzell, S. (1995). Alzheimer and vascular dementias and driving: a prospective road and laboratory study. Journal of the American Medical Association, 273, 1360-1365.

Hagge, R. A. (1994). The California driver performance evaluation project: an evaluation of a new driver licensing road test. Sacramento, CA: California Department of Motor Vehicles.

Hoggarth, P. A., Innes, C. R. H., Dalrymple-Alford, J. C., Severinsen, J. E., \& Jones, R. D. (2010). Comparison of a linear and a non-linear model for using sensory-motor, cognitive, personality, and demographic data to predict driving ability in healthy older adults Accident Analysis and Prevention, 42, 1759-1768.

Hunt, L. A., Murphy, C. F., Carr, D., Duchek, J. M., Buckles, V., \& Morris, J. C. (1997). Reliability of the Washington University Road Test. Archives of Neurology, 54, 707-712.

Janke, M. K., \& Eberhard, J. W. (1998). Assessing medically impaired older drivers in a licensing agency setting. Accident Analysis and Prevention, 30, 347-361.

Korner-Bitensky, N., Bitensky, J., Sofer, S., Man-Son-Hing, M., \& Gelinas, I. (2006). Driving evaluation practices of clinicians working in the United States and Canada. American Journal of Occupational Therapy, 60, 428-434.

Romanowicz, P. A., \& Hagge, R. A. (1995). An evaluation of the validity of California's driving performance evaluation road test. Sacramento, CA: California Department of Motor Vehicles. 